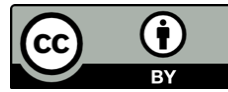

\title{
Percepción social de la inseguridad alimenticia
}

Social perception of food insecurity

Percepção social da insegurança alimentar

\section{ARTÍCULO GENERAL}

\section{Cesar Loo Gil \\ https://orcid.org/0000-0001-8396-5972 \\ cesarloo@biofab.com.pe \\ BioFab Inc, Lima - Perú}

Recibido 05 de Febrero 2021 | Arbitrado y aceptado 19 de Abril 2021 | Publicado el 28 de Julio 2021

\section{RESUMEN}

Este artículo analiza la percepción social de la seguridad alimentaria. Se pretende contribuir a una mejor comprensión de los significados de la seguridad alimentaria, específicamente sobre: riesgos en la alimentación habitual de las familias de bajos ingresos; cambios a largo plazo de su dieta y del contexto; así como de sus esfuerzos para proteger e inclusive mejorar la alimentación familiar. En el medio urbano, esas mujeres perciben riesgos principalmente en la calidad de los alimentos (food safety), mientras que en el medio rural los advierten tanto en el acceso a los alimentos (food security) como en la baja calidad de los mismos. Descubrimos dos rasgos nuevos de la percepción de la seguridad alimentaria: el tiempo disponible del ama de casa urbana para cocinar y la continuidad en el campo de una cultura étnica con saberes y habilidades femeninas valiosas. Aún falta mucho por hacer para alcanzar los diferentes tipos de seguridad alimentaria, así como para contar con una democracia alimentaria plena, al igual que una evolución aceptable en el aspecto nutricional.

Palabras clave: Seguridad, Alimentación, Democracia alimentaria, Significado

\section{ABSTRACT}

This article analyzes the social perception of food security. It aims to contribute to a better understanding of the meanings of food security, specifically about: risks in the usual diet of low-income families; long-term changes in their diet and context; as well as their efforts to protect and even improve family nutrition. In urban areas, these women perceive risks mainly in food quality (food safety), while in rural areas they perceive risks in both food access (food security) and low food quality. We discovered two new features of the perception of food security: the time available for the urban housewife to cook and the continuity in the countryside of an ethnic culture with valuable female knowledge and skills. There is still a long way to go to achieve the different types of food security, as well as full food democracy and an acceptable evolution in the nutritional aspect.

Keywords: Security, Safety, Food, Food Democracy, Meaning

\section{RESUMO}

Este artigo analisa a percepção social da segurança alimentar. Visa contribuir para uma melhor compreensão dos significados da segurança alimentar, especificamente sobre: riscos na alimentação habitual de famílias de baixa renda; mudanças de longo prazo em sua dieta e contexto; bem como seus esforços para proteger e até melhorar a nutrição familiar. Nas áreas urbanas, essas mulheres percebem riscos principalmente na qualidade dos alimentos (segurança alimentar), enquanto nas áreas rurais percebem riscos tanto no acesso aos alimentos (segurança alimentar) quanto na baixa qualidade dos alimentos. Descobrimos duas novas características da percepção de segurança alimentar: o tempo disponível para a dona de casa urbana cozinhar e a continuidade no campo de uma cultura étnica com valiosos conhecimentos e habilidades femininas. Ainda há um longo caminho a percorrer para alcançar os diferentes tipos de segurança alimentar, bem como a plena democracia alimentar e uma evolução aceitável no aspecto nutricional.

Palavras-chave: Segurança, Segurança, Alimentação, Democracia Alimentar, Significado 


\section{Introducción}

La propuesta que hago aquí es estudiar la percepción social de la seguridad y el riesgo alimenticio entre familias de bajos ingresos. Ello exige privilegiar el papel simbólico de los alimentos, por sobre su rol de aporte de nutrientes y de bienes económicos. Eso corresponde a un as- pecto crucial que se ha soslayado en los años recientes: la percepción ordinaria que los pueblos tienen sobre la seguridad y el riesgo en torno a su alimentación cotidiana. Esta percepción social se inserta en el marco de las culturas populares, cuyas características e implicaciones suelen marginarse por los centros de investigación y los organismos internacio- nales y nacionales con políticas en ese terreno.

Aunque el tema de la seguridad alimentaria ha venido recibiendo una creciente atención en los espacios de la opinión pública, los estudios académicos y de las organizaciones sociales, su tratamiento se ha reducido a encuadres muy universalistas y orientados al lado de la producción y abasto. Pero de cualquier modo, en el concepto de seguridad alimentaria se ubican un sentido cuantitativo y otro cualitativo. Para decirlo en los términos con que se nombra este tema en el idioma inglés (Esparza, 2002): con "food security" se alude la dimensión cuantitativa del acceso a los alimentos en general, mientras que "food safety" se usa para aspectos de calidad, como la inocuidad. En los círculos oficiales, de acuerdo a lo señalado por diversos autores, ha prevalecido en la materia un énfasis hacia los aspectos cuantitativos, utilizando parámetros objetivistas, productivistas y mercantilistas, aunque recientemente la Cumbre Mun- dial de Alimentos del 2002 incorporó la necesidad de asegurar la inocuidad y el respeto a las preferencias locales de consumo. Un ejemplo de la vi- sión más convencional de la seguridad alimentaria -referido a la "food security"- lo constituye los estudios que miden niveles de satisfacción de estándares nacionales de consumo, sean éstos promedios calóricoproteicos o el acceso a determinado conjunto de bienes de consumo po- pular (canastas básicas). Así, se analiza (Sampaio y Cardoso, 2002) com- parativamente el consumo de alimentos entre América Latina y la Unión Europea considerando la disponibilidad y consumo de ciertos montos de calorías diarias por habitante (considerando, por ejemplo, tres estratos: bajo $<2,400$ cal., intermedio $>2,400<2,800$ y elevado $<2,800$ ). Esta manera de entender la seguridad alimentaria tiene una relación más o me- nos directa con la nutrición, pues a ésta compete la dimensión de consu- mo alimenticio en términos de 
cantidades de calorías y de proteínas (y su relación con la satisfacción de las necesidades fisiológicas) y el análisis consecuente de los problemas del hambre y la sobrealimentación. Otra asociación entre nutrición y seguridad alimentaria se establece en el ámbito del término "food safety"-al entender a la segunda como la ga- rantía de la calidad sanitaria y nutricional de los alimentos, lo que corresponde, por el lado del riesgo, a los problemas de baja calidad nutricional y de contaminación de los alimentos consumidos por la po- blación. Sin demérito de lo anterior, es conveniente incorporar las visio- nes ordinarias de las poblaciones, las cuales se enmarcan en el terreno de las subjetividades colectivas. Sobre este aspecto es donde resulta relevante el enfoque socioantropológico de la alimentación, es decir, el estudio cultural del comer. Mi propuesta se sitúa del lado de los consumidores, y particularmente, aquellos que tienen bajos ingresos, que constituyen la enorme mayoría de la sociedad en nuestro país y en el mundo subdesarrollado. Comparto el señalamiento (Esparza, 2002) de que el análisis de la seguridad alimentaria desde la perspectiva del consumidor comien- za con un cuestionamiento de las motivaciones de consumo de las perso- nas y del manejo del deseo de éstas, lo que sin duda, se sitúa en el espacio simbólico donde ocurre el fenómeno alimentario.

Trataré de definir en los siguientes términos el concepto de percep- ción social que usaré en el presente artículo. El término percepción es usado en el mundo hispanoamericano tanto para señalar la representa- ción mental de lo captado por los sentidos, como para indicar ideas, co- nocimientos y sensaciones internas. En cuanto a México, el percibir tiene una acepción sensorial, pero también puede tratarse del darse cuenta de algo (como percibir un peligro). Considerando ambos usos, me pa rece que para abordar el tema de la seguridad alimentaria podemos definir al concepto de percepción social como la representación mental de lo captado por los sentidos en la cotidianidad de un grupo social. La dimensión alimenticia entra de manera primordial a la conciencia individual por los sentidos, particularmente por el gusto, aunque también por el olfato, la vista y el tacto. Estas sensaciones se cotejan, vía la memoria, con el arsenal de recuerdos agradables y desagradables que hemos acu- mulado en nuestra experiencia de vida, y a la vez, ellas son reinterpretadas por los saberes, codificaciones y valores relativos a este espacio personal de la existencia. Ahora bien, podemos hablar de una percepción social toda vez que la sensualidad, la emotividad y el conocimiento no quedan confinados en el 
individuo, sino que se establecen afinidades y divergen- cias a nivel familiar, étnico, territorial, etc., surgiendo tipicidades claramente observables tanto a nivel de la sensibilidad como del discurso social, evidenciando a la comida como un hecho cultural de múltiples sig- nificaciones. De este modo, al indagar sobre la percepción social no se trata de verificar la apropiación popular de los aspectos científicos de la ingestión de alimentos, trátese de la calidad o cantidad de ésta. El objeto de conocimiento en el estudio de esa percepción social son las nociones populares, las creencias, expectativas, estereotipos, temores y fervores de los consumidores de alimentos, pensando en ellos en términos de plura- lidad, divergencia y aun de oposiciones. Esta percepción social se conecta con la doxa, ámbito del sentido común, en la cual el discurso social no es producido por los especialistas modernos del campo alimentario (agrónomos, médicos, economistas, empresas agropecuarias y agroindustriales, y cadenas de autoservicio, entre otros), sino por los especialistas tradicionales del campo alimentario, las amas de casa, quienes son una capa clave de los consumidores, en el caso de México y aún entre muchos países del tercer mundo; ellas siguen siendo las responsables únicas o principales de comprar y preparar los alimentos de los núcleos domésticos.

A continuación mencionaré una de las consecuencias negativas del desconocimiento de los procesos culturales colectivos en el tema alimentario. Una de las estudiosas contemporáneas en este problema (Carrasco, 1992), ha argumentado que las políticas de intervención nutricional deberían de superar las dicotomías entre tradición y moder- nidad que, igual en países ricos como pobres, atribuyen sus prácticas de consumo negativas tanto a la persistencia de tradiciones como a los cam- bios modernizadores. Desde su perspectiva, tanto la educación nutricional como la ayuda material alimenticia, deberían de ser complementadas con una adecuada animación sociocultural, la cual debería de incluir las mo tivaciones colectivas del cambio y la continuidad en las prácticas alimentarias.

En la alimentación cotidiana se manifiesta un abanico de expresiones de rechazo, recelo y apego en torno a los diversos productos alimenticios. Frente a ello, mi objetivo en el presente artículo, es efectuar una explora- ción cualitativa del universo de los diversos significados que pudieran existir en el ámbito popular en torno a las actitudes y prácticas de consu- mo alimentario familiar. 


\section{METODOLOGÍA}

La metodología utilizada consta de búsqueda de información de paper, revistas, análisis de aplicaciones actuales y páginas web especializadas para entender su funcionamiento, el nivel de desarrollo obtenido en la actualidad y cuáles son sus principales aplicaciones. Además, se emplean análisis de modelos estratégicos acordes para analizar el atractivo de las industrias en la adopción de la tecnología y así determinar a través de modelos de análisis de negocio cuáles son las industrias en que tendrá mayor incidencia.

La búsqueda de la información para la revisión de literatura se hizo consultando las siguientes bases de datos como son Proquest, Scopus y Google Scholar para encontrar revistas del más alto nivel científico. Asimismo, cabe mencionar que para la realización de lo mencionado se emplearon también palabras clave como como Computación en la nube, Contrato de servicios, Privacidad, ser humano.

Cada uno de estos descriptores se combinaron entre sí durante la búsqueda utilizando los operadores boléanos “and" y “or". Además, algunos de los criterios o filtros que se utilizaron para hacer muchos más específica la búsqueda y ser más precisos con los documentos encontrados en las diferentes bases de datos consultadas fueron: que sean artículos de revistas, y la antigüedad de la publicación que no sea mayor a 5 años.

Toda la información previa relacionada a los filtros aplicados y las especificaciones sirvieron como criterios de inclusión, es decir que sean artículos publicados en revistas científicas, que no excedan los cinco años de antigüedad, que la temática guarde relación con las variables de creatividad artística y tiempos de pandemia. Se excluyeron, en tanto, todos los documentos que no respetaran lo postulado así como aquellos que estaban incompletos o que tenían enlaces averiados.

\section{RESULTADOS Y DISCUSION}

\section{Cuestiones teórico-metodológicas}

Antes de entrar a discutir el problema en sus dimensiones empíricas, conviene explicitar la metodología empleada y repasar algunos plantea- mientos conceptuales relevantes en la literatura de las ciencias sociales y humanas. En este ámbito, he localizado diversas formulaciones teóricas que indirectamente se relacionan con la seguridad alimentaria, y que se refieren a la clasificación cultural de los alimentos, una 
cuestión más amplia donde considero que tiene asidero la primer cuestión. En este tema no existe un consenso, aunque sí algunos puntos convergentes y aún concordantes. Se advierte de inmediato un abanico de posiciones que se extiende desde la posición más optimista (Wenkam), hasta la más es- céptica (Giard), en cuanto a las capacidades humanas de discriminar los alimentos según su grado de riesgo para los propios seres humanos.

Comencemos por Wenkam (1969), quien incorpora un concepto muy novedoso con relación a la discusión internacional sobre seguridad alimentaria: se trata de la disponibilidad cultural o concepto que cada cultura tiene sobre la aceptabilidad de los alimentos. Este autor plantea que, con fundamento o sin él, cada grupo humano clasifica a los produc- tos alimenticios en tres categorías: "comestibles", "dañinos" e "inaceptables". Asimismo, Wenkam señala que es más frecuente que estas distin- ciones cuenten con bases subjetivas, que varíen de una región geográfica a otra y que son susceptibles al cambio con el paso del tiempo; aunque también advierte que esa clasificación, en algunos casos, se basa en crite- rios universales, posiblemente objetivos. Estamos, pues, ante una posi- ción típica de relativismo cultural, el cual ha sido muy influyente en la antropología social. A mi juicio, resulta necesario discutir la plausibili- dad de este esquema de clasificación cultural en el estudio de la percep- ción popular u ordinaria sobre la seguridad alimentaria, puesto que, de una manera $u$ otra, permite visualizar la delimitación de fronteras en torno a lo que cada grupo humano considera como alimento propiamente dicho.

En un sentido opuesto a Wenkam se manifiesta Luce Giard (en: Certeau, 1999), quien hace un rechazo tajante de la sabiduría innata en la elección individual de alimentos. Para esta autora, la composición de un régimen alimentario debe conciliar múltiples y sutiles exigencias nutricionales, mismas que, en el pasado, las sociedades tradicionales no resolvieron plenamente debido al subconsumo, como en el presente tampoco lo han logrado las sociedades modernizadas con su sobreconsumo alimenticio. Giard (Certeau, 1999: 171) agrega que en cualquier medio social que uno encueste, se genera de inmediato "un fabuloso repertorio de sande- ces", el cual aglutina indistintamente "secretos de nodriza, prejuicios sin fundamento, informaciones vagas", situación cognitiva que finalmente tiene su ancla en la "ausencia de regulación interna de comportamientos 
alimentarios del hombre". Respecto a la postura de Giard, encontramos al final que implícitamente acepta la clasificación cultural de los alimen- tos -aunque sobre bases totalmente subjetivas-, sobre todo considerando que para Giard (Certeau, 1999: 172) la comida se somete finalmente a un "sistema de diferencias significativas, coherente mediante sus faltas de lógica".

Otra postura de tipo crítico sobre la percepción popular del riesgo y la seguridad correponde a Jesús Contreras (1993). Según éste, "es la cultu- ra la que crea, entre los seres humanos, el sistema de comunicación que dictamina sobre lo comestible y lo no comestible, sobre lo tóxico o sobre la saciedad. Cada sociedad dispone de unas reglas al respecto, general- mente no escritas" (Contreras, 1993: 14). Para ese autor, tales criterios sobre la conveniencia de los alimentos pueden reflejar tanto necesidades racionales como un etnocentrismo difícil de justificar. En última instan- cia, Contreras cree que el hombre sí dispone de "mecanismos para la regulación de la alimentación, una especie de sabiduría del cuerpo", misma que entró en crisis a la par del aumento de ingresos y de las disponibilidades reales en los países desarrollados, junto con las presiones culturales para consumir más y particularmente, de los alimentos grasos. Resultan interesantes estos planteamientos de Contreras, ya que coinci- den parcialmente con lo sostenido por Wenkam y Bourges en cuanto a la existencia de una "sabiduría popular", además de retomar lo descubierto por Claude Fischler en torno a los trastornos culturales que padece la alimentación en las sociedades más modernas.

Con un enfoque sociobiológico, Fischler (1995) estudió críticamente las formas inapropiadas (o malsanas) de comer en las sociedades moder- nas. Él acuñó el multicitado concepto de gastro-anomie, por el cual se establece que los individuos contemporáneos carecen de sugestiones socioculturales claras de lo que deberían ser sus preferencias alimentarias (cuándo, cómo y qué tanto deberían comer). Así, la selección y el consu- mo de alimentos se están convirtiendo de modo creciente en asuntos per- sonales y no sociales, librándose también de restricciones ecológicas o estacionales. En este sentido, en la alimentación contemporánea, las redes de familiares y no familiares tienen cada vez menos intervención y, por lo tanto, los individuos se hallan cada vez con menos apoyo de esas redes sociales en tan crucial requerimiento de su vida diaria. Entonces ocurre que, los seres humanos, fundamentalmente (h)omnívoros, al care- cer de criterios dignos de 
confianza para tomar tales decisiones, experi- mentan un sentido creciente de ansiedad alimentaria -de aquí se com- prende el vínculo establecido por Fischler con la anomia. Finalmente, cabe anotar que resulta indudable que, lo planteado por Fischler respecto a la aceptabilidad cultural de los alimentos, ha influido en distintos estu- diosos del tema, aunque su atención se dirige casi exclusivamente al com- portamiento alimentario en los países desarrollados.

Finalmente, en Bourges (1990) se observa una postura más optimista que la de Wenkam y coincidente con Contreras en cuanto a la existencia de una sabiduría popular, aunque con cierta cautela. Él reparó en el dete- rioro del poder adquisitivo alimentario que causó la crisis de los años ochenta del siglo XX entre las familias de bajos ingresos en la ciudad de México. Bourges (1990: 27) observó que las familias sustituyeron ciertos productos por otros de composición similar o parecida, pero menos costosos y de menor "prestigio social" [...]. Estos cambios permiten, en principio, man- tener el aporte nutrimental de la dieta con un presupuesto menor.

Al respecto, Bourges considera a estos cambios como "indicadores de la existencia de una 'sabiduría' en las respuestas sociales", aunque luego advierte que no procede confiarse en ella, porque, además de no ser siem- pre acertada, restringe la variedad de la dieta (Bourges, 1990: 27-28). Entre los cambios desafortunados en la dieta de las familias mexicanas en los años ochenta, Bourges señala el uso del café o té en vez de leche, la pasta de trigo en lugar del pescado y mermeladas a cambio de frutas.

Me parece interesante la observación de Bourges sobre la capacidad de las familias para lograr con menos ingreso, un nivel nutricional seme- jante al que había antes del impacto de la crisis económica, pero me resulta difícil aceptar la idea de una "sabiduría" popular. Ante esto, me pregunto si más que "instinto" o "sabiduría", lo que rige el proceso de sustitución de los alimentos es el sistema clasificatorio de los bienes ali- menticios que la población ha heredado y reproducido culturalmente. Un sistema que, soportado en símbolos, valores y representaciones, se actua- liza continuamente en cada cambio del contexto económico, particular- mente en las coyunturas críticas, a las cuales se deben dar respuestas inmediatas, aunque no siempre apropiadas.

Como parte de la herencia colonial española, en México existe una clasificación alimenticia de carácter popular y tradicional referida a cua- lidades térmicas. Desde la 
época colonial, la medicina hispánica introdu- jo la teoría griega de los estados de las cosas (sequía, humedad, frío y calor), que logró un gran influjo en el pensamiento aborigen (Aguirre Beltrán, 1963: 262). Conforme a esta clasificación, las plantas tenían distintas temperaturas, lo que debía tomarse muy en cuenta para su con- sumo durante las etapas de enfermedad o atención especial de la salud.

A su vez, un clásico de la antropología (Douglas, 1973) contribuyó a la mejor comprensión de las ideas occidentales contemporáneas acerca de lo sucio, dominadas casi por completo por el contexto de lo patógeno. Ese texto es muy sugerente y permite entrever la posibilidad de que iden- tifiquemos las clasificaciones que de modo social se construyen sobre la comestibilidad de los alimentos, las que generalmente permanecen invi- sibles a nuestra mirada porque las tomamos como algo evidente, lógico, natural, dado, etc., y no como sistemas que por convención cultural he- mos construido y modificado colectivamente.

Al respecto, también me parece conveniente preguntar por el modo en que los estratos populares pueden pensar y hacer distintas operaciones de reemplazo, combinación o adaptación, ante coyunturas críticas en el campo del abasto y la alimentación; considerando en ello no sólo las fronteras entre lo comestible, dañino e inaceptable, sino entre la saciedad y el hambre y otros esquemas de clasificación aplicados en la vida cotidiana y de modo ordinario.

Mi personal aproximación al tema de la seguridad y riesgo alimentario dentro de la cultura popular en nuestro país se ha basado en procedi- mientos metodológicos que a continuación refiero con brevedad. Creo que debemos reconocer que la elección de una técnica de investigación debe ser lo más apropiada posible a los objetivos de conocimiento de cada investigación (Galindo Cáceres, 1998, pp. 24-25). Por ejemplo, si los propósitos son de interacción, la investigación participativa, el socioanálisis y la investigación-acción son las técnicas indicadas; si pre- tendemos la representación, entonces resulta preferible la encuesta, la cartografía, el análisis de contenido y la heurística; y si nuestro interés se centra en la reflexividad social, entonces lo idóneo son varias técnicas que atienden la relación sujeto/objeto en forma recíproca, este es el caso de: la etnografía, la entrevista a profundidad, la historia oral, el análisis del discurso y los grupos de discusión. En mi caso, considero que la técnica de grupo de discusión (llamada 
también "grupo de enfoque" por los estudiosos estadounidenses) se encuentra a medio camino de la en- cuesta y la investigación-acción, posibilitando una mejor ubicación de los porqués en los comportamientos sociales, así como una mejor intelección de la producción y reinterpretación de los discursos sociales. Esta técnica es propicia para la reflexividad ordinaria. Las sesiones de grupo reproducen elementos del discurso social, o mejor dicho, al nivel del habla en que se interactúa allí, los participantes producen un rico flujo de información social en la forma de discurso. La conversación que se pro- duce en la sesión grupal es el medio por el cual los participantes exponen entre sí, sus representaciones de la realidad, configurándose así un cam- po de sentido(s), un campo semántico. Esta producción metódica del dis- curso, este flujo conversacional inducido, tiene una enorme utilidad para el quehacer antropológico y sociológico, oficios científicos que han enfatizado las técnicas "duras" y convencionales como la etnografía y la encuesta. Es por todo lo anterior que elegí a esta técnica de investiga- ción: para estudiar la percepción de las familias de bajos ingresos en torno a la seguridad y el riesgo alimenticios, cuestión que está más involucrada con el papel simbólico de los alimentos que con su función biológica de nutrientes.

Ahora bien, también es sabido que una buena aplicación de esta técni- ca exige cuidar la integración de los grupos: esto es, que se incorporen a ellos personas con rasgos sociales afines, como la edad, el nivel de ingre- sos y la ocupación. Estos son factores que he tratado de atender en mi trabajo sobre la percepción social de la seguridad alimentaria. Los datos que utilizo en el presente artículo, corresponden a dos grupos de discu- sión o enfoque, los cuales se organizaron en la ciudad de Cuernavaca, Morelos, México, entre 10 mujeres de origen rural principalmente, la mayoría con edades entre cuarenta y cincuenta años, con ocupación de ama de casa la cual combinan con el comercio informal y un bajo nivel de ingresos, y que principalmente proceden de los estados de la República más próximos (Puebla, Guerrero y Edo. de México). Las sedes de ambas sesiones fueron las colonias populares de Villa Santiago (junto al pueblo de Ahuatepec) y en La Lagunilla (próxima al centro histórico). Para congregar al primer grupo, mi apoyo durante el "reclutamiento" fue una terapeuta tradicional con la cual todas tenían alguna relación de amistad, de vecindad y una experiencia común de participación en las llamadas CEBs (comunidades eclesiales de base), desde los años seten- tas; pero ni el grupo ni esa terapeuta tenía conmigo un vínculo previo. En el segundo caso, el recurso empleado por 
mí fue el invitar personalmente a una reunión a las esposas de vecinos y amigos míos en la zona suburba- na donde viví cerca de siete años (entre 1993 y 2002), señoras que tampoco tenían conmigo un trato cotidiano. Las sesiones transcurrieron en un ambiente de mucha confianza y sólo hubo una sesión por cada grupo. En ambas ocasiones, no elaboraré previamente "frases detonadoras" como lo recomiendan algunos autores (Chávez, 2001). Mi experiencia con es- tas sesiones y otras que he realizado con el mismo tema, me indican que resulta difícil escapar a la dinámica habitual de las entrevistas grupales, donde el investigador realiza preguntas y se dirige a los asistentes en lo individual.

Con ambos grupos procedí a grabar las sesiones en cintas de audio con la anuencia de las participantes y luego efectuar personalmente la trans- cripción de todo el material, anotando también silencios, ritmos y tonos de voz. Posteriormente, desmenucé los componentes principales de los textos transcritos, que son las frases u oraciones referidas a los diversos aspectos de la seguridad y el riesgo alimentarios. Estos componentes son, a la vez, los elementos más simples del análisis e interpretación que se efectúa con los datos derivados de la técnica de grupos de discusión. Dicho material fue analizado en tres niveles: individual, cada grupo y sendos grupos, lo que nos va indicando algunas correlaciones entre cier- tas formas de percepción de la seguridad y las características sociales y experiencias de vida de las personas informantes.

Resulta provechoso añadir aquí algunas reflexiones sobre el perfil de las mujeres participantes en las sesiones de grupo y los textos producidos en éstas. Una primera cuestión que resalta es el hecho de que la mayoría de estas mujeres ha migrado a la ciudad de Cuernavaca, después de pasar su infancia, y en algunos casos hasta la juventud, en sus lugares de origen, lo cual posibilita que ellas realicen cotejos en las costumbres y prácticas alimentarias de ambos espacios geográficos. Otro hecho impor- tante es el que las informantes se hallen en una etapa de su vida en la cual han vivido un buen tiempo como responsables de las actividades de coci- na en sus familias y en otros hogares distintos a los de ellas (por el traba- jo doméstico asalariado); esta experiencia les permite una mayor infor- mación y reflexividad sobre la alimentación. Un tercer punto es que, mientras el grupo de mujeres con experiencia en participación en las CEBs se apoyaba en este antecedente, para hacer valoraciones críticas sobre la comida "chatarra", en el otro grupo 
de mujeres -donde algunas eran vendedoras ambulantes de alimentos- se advertía una asociación explícita entre sus conocimientos del oficio y las percepciones de riesgo y seguridad que tenían frente a determinados alimentos, que en forma fre- cuente o eventual, consumen sus familias. En términos generales, se podría aseverar que en el discurso generado por las informantes se detecta que las fronteras percibidas entre lo seguro y lo riesgoso en torno a los alimentos se basa en los hábitos y creencias apropiadas desde la sociali- zación primaria (hasta cerca de los 12 años) y que en la edad adulta son modificados por las experiencias migratorias, ocupacionales y organizativas.

\section{Exploración de la percepción popular en torno a seguridad y riesgo}

\section{alimentario}

Como resultado tentativo del análisis efectuado, presento a continuación de modo conciso, dos configuraciones posibles de los rasgos de la per- cepción popular que esos dos grupos de mujeres mexicanas tienen sobre la seguridad y el riesgo alimentarios. Ambas configuraciones pretenden ir de lo más próximo o sensible, a lo más distante o intangible.

En un plano inicial, los alimentos que consumen y conocen las muje- res de la muestra, ya son materia de clasificación según el riesgo perci- bido en el consumo familiar cotidiano. De inmediato se distinguen dos grupos básicos de alimentos, según las valoraciones que se fueron ha- ciendo a lo largo de las sesiones: el de los productos seguros y el de los riesgosos (véase el siguiente cuadro):

Clasificación de alimentos según el riesgo percibido en el consumo familiar

\begin{tabular}{|l|l|}
\hline Seguros & Riesgosos \\
\hline & Carne de puerco \\
& Bisteces de res \\
& Pescado2 \\
Maíz Frijol Arroz Haba & Mariscos2 \\
Lentejas Garbanzo & Embutidos (salchichas, jamón) \\
Frutas Verduras & Huevo3 (a los niños) \\
Aves de corral (pollo) & Leche (sin refrigerar o cuando no se tiene hábito) Vegetales (sin \\
Quesos naturales & desinfectar) \\
Cecina1 & Pan (de panadería moderna) \\
Carne de chivo (recién & Frijoles instantáneos \\
sacrificado) Yogurt & Moles industrializados \\
Té & Mayonesa \\
& Café2 \\
& Refrescos \\
& Golosinas \\
\hline
\end{tabular}


\begin{tabular}{|l|l}
\hline & (frituras, pastelillos y helados) Alimentos con colorantes
\end{tabular}

Alimentos producidos con agroquímicos

1 Se llega a considerar de riesgo, si no se compra con el debido cuidado y esmero.

2 En unos casos no se considera riesgoso, sobre todo cuando se acostumbró desde pequeño.

3 En unos casos no se considera riesgoso, sobre todo si es producido domésticamente o "de rancho".

\section{CONCLUSIONES}

En este artículo se aborda la percepción social de la seguridad alimentaria entre amas de casa de bajos ingresos del estado de Michoacán, habitantes de localidades rurales e indígenas de la región purhépecha, así como de colonias populares en la ciudad de Morelia. Con un enfoque socioantropológico, se aplicó la técnica de grupos de discusión, con cuatro sesiones definitivas; con base en ellas se ofrece aquí una visión amplia y comparada del universo de representaciones generadas en el seno familiar en el escenario de la seguridad alimentaria en México.

La decisión de estudiar la percepción de la seguridad alimentaria entre familias michoacanas de México ha sido fructífera en tanto que el análisis de grupos de mujeres en distintos contextos -geográfico (rural y urbano) y étnico (indígena y mestizo) - pero en análogas condiciones de pobreza, permite establecer con provecho similitudes y diferencias en esta cuestión vital de su existencia, todo ello según el marco teórico adoptado conforme a nuestro objetivo y planteamiento del problema.

Considerando las cuatro dimensiones de la seguridad alimentaria referi- das en el marco conceptual (Rangel 2002), las mujeres urbanas y mestizas estudiadas perciben riesgos alimentarios en un rango más reducido de tipos o formas. Así, las referencias de los grupos de discusión se centraron en los problemas de calidad sanitaria y nutricional de los alimentos, mencio nándose apenas la problemática de acceso a los alimentos por ingresos insuficientes (se informa que ocurre ocasionalmente). En contraste, las mujeres rurales e indígenas reportaron peligros en tres aspectos (insuficiente producción y abasto, dificultad de acceso a los víveres por insolvencia monetaria y fallas sanitarias y nutricionales), al registrarse sólo un aspec- to con cierta fortaleza (acceso a la base genética productiva), debido a la continuidad y resistencia de la cultura purhépecha. Sin 
embargo, por la condición histórica y actual de la pobreza, las familias indígenas encaran con mayor frecuencia y ciclicidad los riesgos alimentarios.

En cuanto a la calidad nutricional, las mujeres de ambos contextos étnicos y geográficos comparten las dudas y la poca confianza en los comestibles expendidos por el comercio local, especialmente si éstos son "enlatados", golosinas o instantáneos. Finalmente, es relevante el hecho de que, tanto entre unas como otras, la percepción de riesgo alimentario se extiende sobre los productos de la industria agroalimentaria, lo cual nos muestra que, en México, existe población consumidora para la cual los cambios tecnológicos en el sector alimentario no le son indiferentes, como se ha conceptualizado a nivel universal con los consumidores pasivos o reflejo (y esto ocurre aun bajo condiciones de pobreza de ingresos).

En lo relativo a la democracia alimentaria definida por Lang, tenemos una situación análoga de carencia de información, organización del consumidor, elecciones conscientes de compra y, en general, ejercicio de derechos del consumidor. La diferencia estriba, tal vez, en que mientras en el medio urbano la falta de democracia alimentaria se agrava por el clasismo con el que se actúa hacia las familias pobres de la ciudad, en el campo el factor crítico es atribuible al racismo de los comerciantes foráneos.

Los condicionamientos socioculturales referidos por Contreras (el influjo de los usos no nutricionales de los alimentos) intervienen con diferentes sentidos entre las mujeres del campo y de la ciudad. Según la percepción de las mujeres urbanas, su alimentación actual carga con desventajas cul- turales (artificiosidad, simplificación, apremio), aunque simultáneamente advierten muchas alternativas posibles para enfrentar tales desventajas y los riesgos nutricionales, todas ellas en un marco de ingenio, prudencia, sentido común, esfuerzo y amor filial. En el marco de la cultura rural e indígena, se suscitan tanto vergüenza como enojo en relación con el acceso a alimentos básicos y las preferencias de consumo modernas, como gusto y orgullo étnico al comer lo que se cosecha y recolecta por propia mano. Incluso, existe autoreconocimiento explícito del ingenio y de la identidad colectivos al resolver el complejo asunto del sustento diario.

Desde el punto de vista del proceso de transición alimentaria que Méxi- co experimentó desde los años sesenta del siglo XX (Chávez), los sendos grupos de mujeres 
urbanas se expresaron con pesimismo de las transfor- maciones globales de la alimentación en una misma generación de amas de casa (de 1960 al 2000), en tanto que a las mujeres indígenas la seguridad alimentaria familiar les ha parecido precaria por siempre (por la pobreza transgeneracional), fluctuante a lo largo del año (según las estaciones del año) y agravada por la emigración de los jóvenes (con remesas tardías para el sustento de los viejos que se quedan).

Se observan dos facetas no institucionales de la percepción de la segu- ridad alimentaria: la del tiempo disponible del ama de casa para cocinar los alimentos diarios (esto se manifestó sólo en la ciudad) y la existencia de una sólida cultura étnica con saberes y habilidades de que disponen las mujeres para sus responsabilidades domésticas (esto se encontró mucho más en el campo). Es claro que estas dos dimensiones corresponden a la esfera de lo privado, pero que a la vez tienen un claro vínculo con la posición social y con el legado cultural.

Finalmente, cabe señalar que la seguridad alimentaria en la escala fami- liar es un ámbito muy relevante para la intervención del Estado que procura el bienestar común, así como de los grupos sociales (organizaciones de la sociedad civil) y ciudadanía (incluyendo a los investigadores). Es cierto que aún falta mucho por hacer para alcanzar los diferentes tipos de seguridad alimentaria definidos por Rangel, así como una plena democracia alimen- taria, lo mismo que una evolución de México en el campo nutricional y de salud. Pero para orientar mejor las acciones en este campo, no son suficien- tes las encuestas periódicas (nutricionales, de ingreso-gasto): deberíamos atender, también, la percepción de la población sobre su situación. Y para ello pueden servir enfoques como el usado en este artículo.

\section{RECOMENDACIONES}

Aguirre Beltrán, Gonzalo (1963). Medicina y magia. El proceso de aculturación en la estructura colonial. México, Instituto Nacional Indigenista.

Bourges R., Héctor (1990). “Costumbres, prácticas y hábitos alimentarios”, Cua- dernos de Nutrición, Vol. 13, No.2, 3a. Época, año X, México, Fomento de Nutrición y Salud A.C., 17-32 pp.

Certeau, Michel de (1999). La invención de lo cotidiano. 2: Habitar, cocinar. México, Universidad Iberoamericana-ITESO, $271 \mathrm{pp}$. 
Contreras Hernández, Jesús (1993). Antropología de la alimentación. Madrid, Eudema, 96 pp.

Chávez, Adolfo y Miriam (1995). La nueva alimentación. Cómo evitar nuestra desnutrición oculta y el envejecimiento prematuro. México, Procuraduría Federal del Consumidor-Editorial Pax México.

Chávez Méndez, Ma. Guadalupe (2001). De cuerpo entero... Todo por hablar de música. Tesis de doctorado en Ciencias Sociales, México, Universidad de Colima, 344 pp.

Douglas, Mary (1973). Pureza y peligro. Un análisis de los conceptos de contami- nación y tabú. España, Siglo XXI de España Editores, 243 pp.

Esparza, Luis L. (2002). “Globalización y seguridad alimentaria en México”, Po- nencia del XXIV Coloquio de Antropología e Historia Regionales, México, El Colegio de Michoacán, A.C.

Fischler, C. (1995). El (h)omnívoro. El gusto, la cocina y el cuerpo. Barcelona, Anagrama, Col. Argumentos No. 168.

Galindo, J. (1998). Técnicas de investigación en sociedad, cultura y comunica- ción. México, Addison Wesley Longman.

Giddens, Anthony (2000). Un mundo desbocado. México, Taurus, 118 pp. Oseguera Parra, David (2001). Herederos, diversos y conversos. La formación de la cultura alimentaria colimense. Tesis de Doctorado en Antropología Social,

México,

UAM-I., 388

pp.

Pelto, Gretel H. y Pertti J. Pelto (1990). “Dieta y deslocalización: cambios dietéticos desde 1750", en: El hambre en la historia, Rotberg, Robert I. Y rabb,

$\begin{array}{lllll}\text { Theodore } & \text { K. } & \text { (Comps.), } & \text { México, } & \text { Siglo }\end{array}$

Sampaio, M.F.A. y Cardoso, J.L. (2002). “Análise Comparativa do Consumo de Alimentos: América Latina e Uniao Européia”. Brasil, Ponencia al Congreso de ALASRU. Universidad Federal de Rio Grande do Sul.

Torres Torres, Felipe (2001). La alimentación de los mexicanos en la alborada del tercer milenio. México, UNAM-Miguel Ángel Porrúa., 259 pp.

Wenkam, N.S. (1969). Cultural Determinants of Nutritional Behavior. Washington, D.C., Nutrition Program News, U.S.D.A. July-August. 